\title{
DADA: Data Assimilation for the Detection and 2 Attribution of Weather and Climate-related Events
}

\author{
A. Hannart - A. Carrassi - M. Bocquet • \\ M. Ghil • P. Naveau - M. Pulido · J. \\ Ruiz - P. Tandeo
}

7 Received: date / Accepted: date
A. Hannart

IFAECI, CNRS-CONICET-UBA

Pab. II, piso 2, Ciudad Universitaria

1428 Buenos Aires, Argentina

Tel.: +5411-4787-2693

Fax: +5411-4788-3572

E-mail: alexis.hannart@cima.fcen.uba.ar

A. Carrassi

Mohn-Sverdrup Center, Nansen Environmental and Remote Sensing Center, Bergen, Norway

M. Bocquet

CEREA, joint laboratory École des Ponts ParisTech and EDF R\&D, Université Paris-Est, Champs-sur-Marne, France

M. Ghil

Ecole Normale Supérieure, Paris, France

University of California, Los Angeles, USA

P. Naveau

LSCE, CNRS, Gif-sur-Yvette, France

M. Pulido

Dept. of Physics, Universidad Nacional del Nordeste, Corrientes, Argentina

J. Ruiz

IFAECI, CNRS/CONICET/UBA, Buenos Aires, Argentina

P. Tandeo

Télécom Bretagne, Brest, France 
1 relying on data and methods that are routinely available when numerically 12 forecasting the weather. We thus show that causal attribution can be ob3 tained as a by-product of data assimilation procedures run on a daily basis to update numerical weather prediction (NWP) models with new atmospheric observations; hence, the proposed methodology can take advantage of the powerful computational and observational capacity of weather forecasting centers. We explain the theoretical rationale of this approach and sketch the most prominent features of a "data assimilation-based detection and attribution" (DADA) procedure. The proposal is illustrated in the context of the classical three-variable Lorenz model with additional forcing. The paper concludes by raising several theoretical and practical questions that need to be addressed to make the proposal operational within NWP centers.

Keywords Event attribution · Data assimilation · Causality theory • Modified Lorenz model

\section{Background and motivation}

Providing causal assessments about episodes of extreme weather or unusual climate conditions is an important topic in the climate sciences: it arises from the multiple needs for public dissemination, litigation in a legal context, adaptation to climate change or simply improvement of the science associated with these events (Stott et al., 2013). The approach widely used so far to was introduced one decade ago by M.R. Allen and colleagues (Allen, 2003; Stone and Allen, 2005); it originates from best practices in epidemiology (Greenland and Rothman, 1998) and is referred to as probabilistic event attribution (PEA).

In the PEA approach, one evaluates the extent to which a given external climate forcing - such as solar irradiation, greenhouse gas (GHG) emissions, ozone or aerosol concentrations - has changed the probability of occurrence of an event of interest. For this purpose, one thus needs to compute two probabilities: (i) the probability of occurrence of the event in an ensemble of model simulations representing the observed climatic conditions, which simulates the actual occurrence probability in the real world, referred to as factual; and (ii) the probability of occurrence of the event in a second ensemble of model simulations, representing this time the alternative world that might have occurred had the forcing of interest been absent, referred to as counterfactual.

Denoting by $p_{1}$ and $p_{0}$ the probabilities of the event occurring in the factual world and in the counterfactual world respectively, the so-called fraction of attributable risk (FAR) is then defined:

$$
\mathrm{FAR}=1-\frac{p_{0}}{p_{1}}
$$

The FAR has long been interpreted as the fraction of the change in likelihood of an event which is attributable to the external forcing. Over the past decade, most causal claims have been following from the FAR and its uncertainty, resulting in statements such as "It is very likely that over half the risk of 
European summer temperature anomalies exceeding a threshold of $1.6^{\circ} \mathrm{C}$ is attributable to human influence." (Stott et al., 2004). Hannart et al. (2015) have recently shown that, under realistic assumptions, the FAR may also be interpreted as the so-called probability of necessary causation $(\mathrm{PN})$ associated — in a complete and self-consistent theory of causality (Pearl, 2000) — with the causal link between the forcing and the event. The FAR thus corresponds to only one of the two facets of causality in such a theory, while the probability of sufficient causation (PS) is its second facet.

In this setting,

$$
\begin{aligned}
& \mathrm{PN}=1-\frac{p_{0}}{p_{1}}, \\
& \mathrm{PS}=1-\frac{1-p_{1}}{1-p_{0}}, \\
& \mathrm{PNS}=p_{1}-p_{0},
\end{aligned}
$$

where PNS is the probability of necessary and sufficient causation. Pearl (2000) provides rigorous definitions of these three concepts, as well as a detailed discussion of their meanings and implications. It can be seen from Eqs. (2) that causal attribution requires to evaluate the two probabilities, $p_{0}$ and $p_{1}$, which is therefore the central methodological question of PEA.

So far, most case studies have used large ensembles of climate model simulations in order to estimate $p_{1}$ and $p_{0}$ based on a variety of methods. However, this general approach has a very high computational cost and is difficult to implement in a timely and systematic way. As recognized by Stott et al. (2013), this remains an open problem: "the overarching challenge for the community is to move beyond research-mode case studies and to develop systems that can deliver regular, reliable and timely assessments in the aftermath of notable weather and climate-related events, typically in the weeks or months following (and not many years later as is the case with some research-mode studies)". Several research initiatives are presently addressing this real-time attribution challenge. For instance, the weather@home system (Massey et al., 2014) in the context of the World Weather Attribution initiative (http://www.climatecentral.org/wwa), the system proposed by Christidis et al. (2013), or the Weather Risk Attribution Forecast system (http://www.csag.uct.ac.za/ daithi/forecast/) aim at meeting those requirements within the conventional ensemble-based approach.

The purpose of this article is to introduce a new methodological approach that addresses the latter overarching operational challenge. Our proposal relies on a class of powerful statistical methods for interfacing high-dimensional models with large observational datasets. This class of methods originates from the field of weather forecasting and is referred to as data assimilation (DA) (Bengtsson et al., 1981; Ghil and Malanotte-Rizzoli, 1991; Talagrand, 1997).

Section 2 explains the rationale of the approach proposed herein, presents a brief overview of DA, and outlines the most prominent technical features of a "data assimilation-based detection and attribution" (DADA) approach. 
Section 3 illustrates the proposal by implementing it on a version of the classical Lorenz convection model (Lorenz, 1963, L63 hereafter) subject to an additional constant force. Finally, in Section 4, we discuss the main strengths and limitations of the DADA approach, and highlight several theoretical and practical research questions that need to be addressed to make it potentially operational within weather forecasting centers in a near future.

\section{Methodology}

\subsection{General rationale}

In an operational context, a significant difficulty of PEA is that events of interest are usually rare, i.e. they occur in regions of the climate system's attractor that are reached quite rarely. It may hence require a very large ensemble of simulations for the numerical model representing the climate system to reach the relevant region of the attractor. This requirement is particularly relevant if the event is defined narrowly, based on multiple features that might involve some combination of the atmospheric circulation, of the climate system's thermodynamic state, and of the impacts associated with the event. Simulating a sufficiently large number of occurrences of such an event for a robust evaluation of $p_{1}$ and $p_{0}$ may then be computationally very costly, and a brute force approach based on an unconstrained ensemble may become unaffordable in an operational context.

The first general idea underlying the DADA proposal is that the latter computational burden may be greatly reduced by constraining the model to explore only the relevant region of its state space where the event under scrutiny is defined to occur. Such a selective exploration of a high-dimensional state space is not new. The constrained simulation of very rare events using complex dynamical models has been studied extensively (e.g., Harris and Kahn (1951); Del Moral and Garnier (2005)) and is referred to as Rare Event Sampling (RES). RES methods are based on importance sampling and probabilistic large-deviation theory (Bucklew, 2004), and they are commonly used in several areas - such as queueing, reliability, telecommunication (Heidelberg, 1995) - but their adaptation to a climate context has only recently started (Wouters and Bouchet, 2015).

The second general idea of the DADA proposal is to take a shortcut along this path: DA methods present the key advantage of being already operational in weather forecasting centers to routinely update an atmospheric model with new observations in order to initialize the forecast, and we argue that they can be used simultaneously to solve the class of problems addressed by RES methods. Carrassi et al. (2008, and references therein) have already used a similarly selective exploration of a reduced number of phase space dimensions in the context of DA methods designed to control chaotic dynamics.

For the purposes of PEA, we show that,by assimilating the observed trajectory of an event into a model, one can obtain as a by-product the probability 
density function (PDF) associated with this trajectory. PEA is then obtained by assimilating the observations of the event twice, first in the factual setting of the model and second in its counterfactual setting, and then by computing the FAR as the ratio of the two PDF values thus obtained.

Heuristically speaking, if an observed event is incompatible with the counterfactual world but compatible with the factual one - according to the standard approach of defining the existence of a causal link (Pearl, 2000; Hannart et al., 2015) - then assimilation will act as a crucial experiment, since the event's observed trajectory will be easy to assimilate in the factual setting and difficult to assimilate in the counterfactual one, merely because the counterfactual setting physically precludes the existence of such a trajectory.

In Subsection 2.2, we formulate this general rationale in probabilistic terms and discuss the relevance of the approach. We then show in Subsection 2.3 that, given a similar set of hypotheses as those that underly the majority of operational DA methods, it is possible to quantify the extent to which an observed trajectory is compatible with the model physics - either factual or counterfactual - or not. This quantification in an operational context is at the core of the DADA approach and it greatly facilitates real-time PEA.

\subsection{Probabilistic description of the method}

Let $\mathbf{y}_{t}$ denote the $d$-dimensional vector of observations at discrete times $\{t=$ $0,1, \ldots, T\}$. Here, $\mathbf{y}=\left\{\mathbf{y}_{t}: 0 \leq t \leq T\right\}$ corresponds, for instance, to the full set of all available meteorological observations over a time interval covering the event of interest, no matter the diversity and source of the data; typically, the latter include ground station networks, satellite measurements, ship data, and so on, cf. Bengtsson et al. (1981, Preface, Fig. 1) or Ghil and MalanotteRizzoli (1991, Fig. 1). In the present probabilistic context of PEA, the observed trajectory $\mathbf{y}$ is viewed as a realization of a random variable denoted $\mathbf{Y}=\left\{\mathbf{Y}_{t}\right.$ : $0 \leq t \leq T\}$, i.e. there exists an $\omega \in \Omega$ such that $\mathbf{Y}(\omega)=\mathbf{y}$ - where $\Omega$ denotes the sample space of all possible outcomes and encompasses observational error, as well as internal variability.

In event attribution studies, it is recognized that defining the occurrence of an event, i.e. selecting a subset $\mathcal{F} \subset \Omega$, depends on a rather arbitrary choice. Yet this choice has been shown to greatly affect causal conclusions (Hannart et al., 2015). For instance, a generic and fairly loose event definition is arguably prone to yield a low level of evidence with respect to both necessary and sufficient causality while, on the other hand, a tighter and more specific event definition is prone to yield a stringent level for necessary causality but a reduced one for sufficient causality.

Indeed, it is quite intuitive that many different factors should usually be necessary to trigger the occurrence of a highly specific event and conversely, that no single factor will ever hold as a sufficient explanation thereof. For the class of unusual events at stake in PEA, where both $p_{0}$ and $p_{1}$ are very small, we arguably lean towards specific definitions that inherently result in 
few sufficient causal factors or none. This conclusion immediately follows from Eq. (1b), which yields $\mathrm{PS} \simeq 0$ when both $p_{0}$ and $p_{1}$ are very small.

Usually, an event occurrence is defined in PEA based on an ad hoc scalar index $\phi(\mathbf{Y})$ exceeding a threshold $u$, i.e. $p_{i}=P(\phi(\mathbf{Y}) \geq u)$; from now on, we associate $i=0$ with the counterfactual and $i=1$ with the factual world. While this definition may be already quite restrictive for $u$ large, it is defensible to restrict the event definition even further. Such a strategy may reduce an already negligible PS but it also may increase PN by a greater amount; one thus expects to gain more than is lost in this trade-off. In particular, this will be the case if additional features, not accounted for in $\phi(\mathbf{Y})$, can be identified that will allow one to further discriminate between the two worlds.

Following this strategy, a central element of our proposal is to use the tightest possible event occurrence definition, i.e. the trajectory $\mathbf{y}$ exactly as it was observed, namely the singleton event $\{\mathbf{Y}=\mathbf{y}\}$. This singleton event has probability zero in both worlds, i.e. $p_{1}=p_{0}=0$. Indeed, the full sequence of observations y, exactly as it occurred, is unique. Quoting the Greek philosopher Heraclitus "You cannot step into the same river twice, for other waters are continually flowing in": the exact same sequence $\mathbf{y}$ never occurred before and will never occur again. Our proposed singleton event definition may thus arguably match with the suggestion of Trenberth et al. (2015) that "a different framing is desirable which asks why extremes unfold the way they do" in so far as it focuses on the event exactly as it happened and is thereby able to spot the detailed physical features of the event that made it "unfold the way it did". However, by contrast with Trenberth et al. (2015), our proposed singleton event definition is not conditional on the circulation: the observed vector $\mathbf{y}$ may perfectly include circulation-related observations.

One may find surprising that a causal analysis of such a zero probability event is possible. However, in the context of the aforementioned causal theory, such a causal analysis is definitely possible and meaningful. Indeed, the fact that $p_{1}$ and $p_{0}$ are null does not imply that the associated probability of necessary causation PN is null. Generally speaking, the ratio of two quantities that tends to zero may well converge to a finite quantity (e.g. the derivative of a differentiable function). Likewise, here the singleton set $\{\mathbf{Y}=\mathbf{y}\}$ may be viewed as the limit of the sphere of radius $r$ centered in $\mathbf{y}$ when the radius $r$ tends to zero, i.e. $\{\mathbf{Y}=\mathbf{y}\}=\lim _{r \rightarrow 0}\{\|\mathbf{Y}-\mathbf{y}\| \leq r\}$. It is clear that when $r \rightarrow 0$, then $p_{0} \rightarrow 0$ and $p_{1} \rightarrow 0$. It is also straightforward to show that the limit of $\mathrm{PN}=1-p_{0} / p_{1}$ is then finite. More specifically, we have:

$$
\mathrm{PN}=1-\frac{f_{0}(\mathbf{y})}{f_{1}(\mathbf{y})}
$$

where we denote $f_{i}$ the PDF of $\mathbf{Y}$ in world $i$. By contrast, the quantity $1-(1-$ $\left.p_{1}\right) /\left(1-p_{0}\right)$ converges to zero when $p_{0}$ and $p_{1}$ tends to zero, thus the probability of sufficient causation PS associated with the singleton event $\{\mathbf{Y}=\mathbf{y}\}$ is always zero. Our DADA proposal thus intentionally sacrifices the evidence of sufficiency, in the hope of maximizing the evidence of necessity. 
Our betting on the singleton set is thus justifiable already based on the above theoretical considerations. This choice, moreover, is also motivated by having a highly simplifying implication from a practical standpoint. Evaluating the PDF of $\mathbf{Y}$ at a single point $\mathbf{Y}=\mathbf{y}$ is indeed, under many circumstances, considerably easier than evaluating the probability $P(\phi(\mathbf{Y}) \geq u$ ) required in the conventional approach. Appendix A gives a concrete illustration of this situation, and Figure 1 shows the details of the latter evaluation for a scalar $\operatorname{AR}(1)$ process (panel $a$, as well as its associated accuracy (panels $b$ and $c$ ), and the computational cost as the sample size $n$ varies (panel $d$ ); the latter cost is much larger than the one of applying the DADA approach consisting in evaluating the PDF at a single point. This simple example confirms the large computational discrepancy between the two approaches. The reason for the discrepancy is quite simple: evaluating the conventional probability requires integrating a PDF over a predefined domain, instead of a one-off evaluation at a single point. Because both the domain of integration and the PDF may have potentially complex shapes, one cannot expect, in general, that the requisite integral be amenable to analytical treatment. Hence numerical integration is the default option: no matter how efficient an integration scheme one applies, it will require evaluating the PDF at many points and is thus as many times more costly computationally than just evaluating $f(\mathbf{y})$ at a single point.

In order to obtain the PDF of $\mathbf{Y}$, the class of dynamic, statistical models referred to as Hidden Markov Models (HMMs; e.g. Ihler et al. (2007)) is relevant in the context of PEA. Indeed, the dynamics of a climate event can usually be represented by using a numerical climate model. Denoting $\mathbf{X}_{t}$ the $N$-dimensional state vector at time $t$ of the numerical model, we can assume:

$$
\begin{aligned}
& \mathbf{X}_{t+1}=\mathrm{M}\left(\mathbf{X}_{t}, \mathbf{F}_{t}\right)+\mathbf{v}_{t}, \\
& \mathbf{Y}_{t}=\mathrm{H}\left(\mathbf{X}_{t}\right)+\mathbf{w}_{t}
\end{aligned}
$$

where Equation (4a) describes the dynamics of the state vector, with $\mathrm{M}$ the numerical model operator, $\mathbf{v}_{t}$ a stochastic term representing modeling error, and $\mathbf{F}_{t}$ a prescribed forcing. Equation (4b) maps the state vector $\mathbf{X}_{t}$ to our observations $\mathbf{Y}_{t}$ at any time $t$, where $\mathrm{H}$ is the so-called observation or forward operator and $\mathbf{w}_{t}$ is a stochastic term representing observational error. The problem of interest here is thus to derive the likelihoods $f_{0}(\mathbf{y})$ and $f_{1}(\mathbf{y})$ of the observation $\mathbf{y}$ when using the counterfactual and factual forcings, by using the HMM setting of Equation (4).

DA can be viewed as a class of inference methods designed for the above HMM setting. While inferring the unknown state vector trajectory $\mathbf{X}$ given the observed trajectory $\mathbf{y}$ is the main focus of DA, the likelihood $f(\mathbf{y})$ can also be obtained as a side product thereof, as we will immediately clarify below. Therefore, with DA able to derive the two likelihoods $f_{0}(\mathbf{y})$ and $f_{1}(\mathbf{y})$, and the latter two being the keys to causal attribution in our approach, one should be capable of moving towards near-real-time, systematic causal attribution of weather- and climate-related events. 
2.3 Brief overview of data assimilation

DA was initially developed in the context of numerical weather forecasting, in order to initialize the model's state variables $\mathbf{X}$ based on observations $\mathbf{y}$ that are incomplete, diverse, unevenly distributed in space and time and are contaminated by measurement error (Bengtsson et al., 1981; Talagrand, 1997). Over the past decades, those methods have grown out of their original application field to reach a wide variety of topics in geophysics such as oceanography (Ghil and Malanotte-Rizzoli, 1991), atmospheric chemistry, geomagnetism, hydrology, and space physics, among many other areas (Robert et al., 2006; Cosme et al., 2010; Kondrashov et al., 2011; Bocquet, 2012; Martin et al., 2014).

DA is already playing an increasing role in the climate sciences, having being applied, for instance, to initialize a climate model for seasonal or decadal prediction (Balmaseda et al., 2009), to constrain a climate model's parameters (Kondrashov et al., 2008; Ruiz et al., 2013), to infer carbon cycle fluxes from atmospheric concentrations (Chevallier, 2013), or to reconstruct paleoclimatic fields out of sparse and indirect observations (Bhend et al., 2012; Roques et al., 2014). In the context of D\&A, Lee et al. (2008) actually tested a DA-like approach to include the effects of the various forcings over the last millennium, in addition to other paleoclimate proxy data, in combined climate reconstruction and detection analysis. The present work thus follows a general trend in climate studies.

Methodologically speaking, DA methods are traditionally grouped into two categories: sequential and variational (Ide et al., 1997, and references therein). Here, we concentrate on the sequential approach, but the two approaches are complementary and the choice of method depends on the specifics of the problem at hand (Ghil and Malanotte-Rizzoli, 1991; Ide et al., 1997; Talagrand, 1997). In the sequential approach (Ghil et al., 1981), the state estimate and a suitable estimate of the associated error covariance matrix are propagated in time until new observations become available and are used to update the state estimate. In practice, the evolution of the system of interest is retrieved - like in earlier, typically much smaller-dimensional applications (Kalman, 1960; Jazwinski, 1970; Gelb, 1974) — through a sequence of prediction and analysis steps.

Abundant literature is available on DA and on Kalman-type filters. Kalman (1960) first presented the solution in discrete time for the case in which both the dynamic evolution operator $\mathrm{M}$ in Eq. (4a) and the observation operator $\mathrm{H}$ in Eq. (4b) are linear, and the errors are Gaussian. Under these assumptions, the state-estimation problem for the system given by Eqs. (4a, 4b) has an exact solution given by the sequential Kalman filter $(\mathrm{KF})$ equations (Appendix B). Further, the likelihood function $f(\mathbf{y})$, which is of primary importance for DADA, also has an exact expression under the above linearity and Gaussianity assumptions (Tandeo et al., 2014). Following the usual notations of DA, which 
$344 \quad \frac{\mathrm{d} x}{\mathrm{~d} t}=\sigma(y-x)+\lambda_{i} \cos \theta_{i}, \quad \frac{\mathrm{d} y}{\mathrm{~d} t}=\rho x-y-x z+\lambda_{i} \sin \theta_{i}, \quad \frac{\mathrm{d} z}{\mathrm{~d} t}=x y-\beta z$ are detailed in Appendix B, the expression of the likelihood is given by:

$$
f(\mathbf{y})=\prod_{t=0}^{T}(2 \pi)^{-\frac{d}{2}}\left|\boldsymbol{\Sigma}_{t}\right|^{-\frac{1}{2}} \exp \left\{-\frac{1}{2}\left(\mathbf{y}_{t}-\mathbf{H} \mathbf{x}_{t}^{f}\right)^{\prime} \boldsymbol{\Sigma}_{t}^{-1}\left(\mathbf{y}_{t}-\mathbf{H} \mathbf{x}_{t}^{f}\right)\right\}
$$

with $\boldsymbol{\Sigma}_{t}=\mathbf{H} \mathbf{P}_{t}^{f} \mathbf{H}^{\prime}+\mathbf{R}$. The proof of Eq. (5) is provided in Appendix C, and $f(\mathbf{y})$ is typically computed by taking the logarithm of this equation to turn the product on the right-hand side into a sum.

The main interest of Eq. (5) is that, once the observations $\mathbf{y}_{t}$ have been assimilated on the interval $0 \leq t \leq T$, the necessary ingredients $\mathbf{x}_{t}^{f}$ and $\mathbf{P}_{t}^{f}$ in Eq. (5) are available from the KF equations (Appendix B) and thus calculating $f(\mathbf{y})$ is both straightforward and computationally inexpensive. The fundamental connections between this calculation, the HMM context, and Bayes theorem are further clarified in Appendix C.

Many difficulties arise in applying the simple ideas outlined here to geophysical models, which are typically nonlinear, have non-Gaussian errors and are huge in size (Ghil and Malanotte-Rizzoli, 1991). Most of these difficulties have been addressed by improving both sequential and variational methods in several ingenious ways (Bocquet et al., 2010; Kondrashov et al., 2011).

In particular, the Ensemble Kalman Filter (EnKF; Evensen, 2003) - in which the uncertainty propagation is evaluated by using a finite-size ensemble of trajectories - is now operational in numerical weather and oceanic prediction centers worldwide; see e.g. Houtekamer et al. (2005); Sakov et al. (2013). The EnKF is a convenient approximate solution to the filtering problem in a nonlinear, large-dimensional context. We simply note here that it can also be applied to obtain an approximation of the likelihood $f(\mathbf{y})$ by substituting the approximate sequence $\left\{\left(\hat{\mathbf{x}}_{t}^{f}, \hat{\mathbf{P}}_{t}^{f}\right): t=0, \ldots, T\right\}$ that the EnKF produces into Eq. (5). This strategy is illustrated immediately below in the context of the L63 convection model subject to an additional constant force.

\section{Implementation within the modified L63 model}

\subsection{The modified model and its two worlds}

A simple modification (Palmer, 1999) of the L63 model (Lorenz, 1963) has been extensively used for the purpose of illustrating methodological developments in both DA and PEA (e.g. Carrassi and Vannitsem, 2010; Stone and Allen, 2005). In the nonlinear, coupled system of three ordinary differential equations (ODEs) for $x, y$ and $z$ below,

the time-constant forcing terms in the $x$ - and $y$-equation represent, in fact, an addition to the forcing hidden in the original L63 model. The latter forcing 
is revealed by a well-known linear change of variables, in which $x$ and $y$ are left unchanged and $z \rightarrow z+\rho+\sigma$ (Lorenz, 1963). In the new variables, the model of Eq. (6) will take the canonical form of a forced-dissipative system (Ghil and Childress, 1987, Sec. 5.4), with an extra forcing term $-\beta(\rho+\sigma)$ in the $z$-equation, just like the original L63 model.

Here $\lambda_{i}$ is the intensity of the additional forcing and $\theta_{i}$ is its direction in world $i=0,1$ : i.e., $\lambda_{0}=0$ represents a counterfactual world with no additional forcing, while $\lambda_{1} \neq 0$. We take the parameters $(\sigma, \rho, \beta)$ to equal their usual values $(10,28,8 / 3)$ that yield the well-known chaotic behavior, and the (nondimensional) time unit $t$ is interpreted as equaling days.

The ODE system given by (6) is discretized by using $\Delta t=0.01$ and $t$ refers hereafter to the number of time increments $\Delta t$. This system is then turned into a HMM as described in Equation (4) by adding an error term $\mathbf{v}_{t}$ assumed to be Gaussian and centered with covariance $\mathbf{Q}=\sigma_{Q}^{2} \mathbf{I}$, where $\mathbf{I}$ is the $3 \times 3$ identity matrix. Furthermore, we assume that all three coordinates $(x, y, z)$ of the state vector are observed, i.e. that $\mathrm{H}=\mathbf{I}$, and that the measurement error term $\mathbf{w}_{t}$ is also Gaussian and centered, with covariance $\mathbf{R}=\sigma_{R}^{2} \mathbf{I}$.

The HMM defined above is stationary, i.e. the PDF of the observed vector $\mathbf{y}_{t}$ depends neither on $t$ nor on the initial condition after a sufficiently long time $t$ (Appendix D). In the factual world, the shape of the PDF is affected by the parameters $\left(\lambda_{1}, \theta_{1}\right)$ of the forcing. In both worlds, the PDFs can be estimated, for instance, by using kernel density estimation applied to ensembles of simulations obtained for either forcing. In Figs. $2 \mathrm{a}, \mathrm{b}$, we plot the projections of both PDFs onto the plane associated with the greatest variance in the factual PDF. The difference between the two PDFs is shown in Fig. 2c; it emphasizes the existence of an area of the state space (represented in white), which is more likely to be reached in the factual world than in the counterfactual one.

Next, we define an event to occur for the sequence $\left\{\mathbf{y}_{t}: t=0, \ldots, T\right\}$ if the scalar product $\hat{\phi}^{\prime} \mathbf{y}_{t}$ between the unit vector $\hat{\phi}$ in the direction $\phi$ and $\mathbf{y}_{t}$, i.e. the projection of $\mathbf{y}_{t}$ onto the direction $\phi$, exceeds $u$ for some $0 \leq t \leq T$, where $\phi$ is a specified direction and $u$ is a threshold chosen based on $\phi$ so that $p_{1}=0.01$. Figure $2 \mathrm{~d}$ shows a selection of sequences from both worlds in which an event did occur, where $\phi$ was chosen to be the leading direction in the projection plane.

For this choice of $\phi$, the trajectories associated with event occurrence happen to all lie in the area of the state space which is more likely to be reached in the factual world than in the counterfactual one. Accordingly, the probability of the event in the former is found to be higher than in the latter, i.e. $p_{1}>p_{0}$, and the occurrence of an event $\left\{\max _{\{0 \leq t \leq T\}} \phi^{\prime} \mathbf{y}_{t} \geq u\right\}$ is thereby informative from a causal perspective.

Figure 2d also shows that the trajectories associated with the event in the two worlds - counterfactual (green) and factual (red) -- appear to have slightly distinct features: the red trajectories are shifted towards higher values in the second direction, of highest-but-one variance. Such distinctions might help discriminate further between the two worlds in the DADA framework - 
the circumstances under which such further discrimination is helpful will be discussed in Section 4.

\subsection{DADA for the modified L63 model}

The DADA procedure is illustrated in Fig. 3. We plot in panel (a) a trajectory of the state vector $\mathbf{x}_{t}$ simulated under factual conditions, i.e. in the presence of the additional forcing (black solid line), along with the observations $\left\{\mathbf{y}_{t}\right.$ : $0 \leq t \leq T\}$ (gray dots), with $T=400$. The EnKF is used to assimilate these observations into a factual model $(i=1)$ that thus matches the true model $\mathrm{M}=\mathrm{M}_{1}=\mathrm{M}\left(\lambda_{1}, \theta_{1}\right)$ used for the simulation: a reconstructed trajectory is obtained from the corresponding analyses $\mathbf{x}_{t}^{a}$ (red solid line in panel (a)), cf. Eqs. (8), and the likelihoods $f_{1}\left(\mathbf{y}_{t}\right)$ (red solid line in panel (c)) are obtained by application of Eq. (5), respectively.

Next, the assimilation is repeated in the counterfactual model $(i=0$, i.e. $\lambda=0$ ) to obtain a second analysis of the trajectory, from the same observations; see green solid line in panel (a), for $T=400$. The corresponding likelihoods $f_{0}\left(\mathbf{y}_{t}\right)$ are shown in panel (c) as a green solid line. Comparing the trajectories of the two analyses in Fig. 3a shows that, even though the counterfactual analysis (green line) uses the same data as the factual analysis (red line), the former lies closer to the true trajectory (black line).

The local discrepancies between the trajectories estimated in the two worlds appear to be rather small at first glance, cf. panel (a), and so are the instantaneous differences between the associated factors on the right-hand side of Eq. (5); the latter are shown as gray rectangles in panel (c) of the figure. Still, the evidence in favor of the factual world accumulates as the time $t$ over which the two trajectories differ, albeit by a small amount, lengthens. This cumulative difference in evidence, $\log f_{0}\left(\mathbf{y}_{t}\right)-\log f_{1}\left(\mathbf{y}_{t}\right)$, is reflected by a growing gap between the two curves, red and green, in panel (c), and by an associated high mean growth over time of the probability PN of necessary causation, cf. the black solid line in panel (d).

In order to evaluate more systematically its performance and robustness compared to the conventional FAR approach, the DADA procedure was applied to a large sample of sequences $\mathbf{y}_{t}$ of length $T=20$ simulated under diverse conditions. The sample explored all possible combinations of the triplet of parameters $\left(\lambda_{1}, \sigma_{Q}, \sigma_{R}\right)$, with ten equidistributed values each, for a total of $10^{3}$ combinations; the ranges were $0 \leq \lambda_{1} \leq 40,0.1 \leq \sigma_{Q} \leq 0.5$ and $0.1 \leq \sigma_{R} \leq 1.0$, respectively, with $\theta_{1}=-140^{\circ}$. For each combination of $\left(\lambda_{1}, \sigma_{Q}, \sigma_{R}\right)$, ten directions $\phi$ were randomly generated and $u$ was defined based on $\phi$ as in Sec. 3a above, so as to achieve $p_{1} \geq 0.01$.

In order to estimate the corresponding conventional probabilities $p_{0}$ and $p_{1}$ of the associated event defined as $\left\{\max _{\{0<t<T\}} \phi^{\prime} \mathbf{y}_{t} \geq u\right\}, n=50000$ sequences $\mathbf{y}_{t}$ of length $T=20$ were simulated, by using a single sequence of length $n T=10^{6}$ and splitting it into $n$ equal segments. Probabilities $p_{0}$ and $p_{1}$ were then directly estimated from empirical frequencies. 
For each quintuplet of parameter values $\left(\lambda_{1}, \sigma_{Q}, \sigma_{R} ; \phi, u\right)$, one hundred sequences of observations $\left\{\mathbf{y}_{t}: 0, \ldots, T=20\right\}$ were generated with a proportion $p_{1} /\left(p_{1}+p_{0}\right)$ being simulated from the factual world and a proportion $p_{0} /\left(p_{1}+p_{0}\right)$ from the counterfactual one. All sequences were treated with the DADA procedure - by applying DA to the synthetic observations according to Eqs. (8a)-(8d) - and then Eq. (5) to obtain $f_{0}(\mathbf{y})$ and $f_{1}(\mathbf{y})$ from the reconstructed trajectories. The a priori mean and covariance $\mathbf{x}_{0}^{f}$ and $\mathbf{P}_{0}^{f}$ required as inputs to the DADA procedure were those associated with the PDF of the attractor, given the forcing conditions $\left(\lambda_{1} \in[0,40], \theta_{1}=-140^{\circ}\right)$ assumed for each assimilation experiment. As a result, two probabilities PN of necessity are finally obtained for each sequence $\mathbf{y}_{t}, \mathrm{PN}_{p}=1-p_{0} / p_{1}$ for the conventional approach and $\mathrm{PN}_{f}=1-f_{0}(\mathbf{y}) / f_{1}(\mathbf{y})$ for the DADA approach.

We next wish to evaluate under various conditions how well the two probabilities $\mathrm{PN}_{p}$ and $\mathrm{PN}_{f}$ perform with respect to discriminating between the factual and counterfactual forcings. Consider a simple discrimination rule whereby a trajectory $\mathbf{y}_{t}$ is identified as factual for $\mathrm{PN}$ exceeding a given threshold, and as counterfactual otherwise. The so-called receiver operating characteristic (ROC) curve plots the rate of true positives as a function of the rate of false positives obtained when varying the threshold in a binary classification scheme from 0 to 1 ; it thus gives an overall visual representation of the skill of our PN as a discriminative score.

The Gini (1921) index $G$ was originally introduced as a measure of statistical dispersion intended to summarize the information contained in the Lorenz (1905) curve that represents the income distribution of a nation's residents; $G$ may be viewed, though, more generally as a metric summarizing the dispersion of any smooth curve that starts at the origin and ends at the point $(1,1)$ with respect to the diagonal of the corresponding square. In particular, we use $G$ here to summarize into a single scalar the ROC curve, which ranges from 0 for random discrimination to 1 for perfect discrimination.

Figure 4a shows ROC curves obtained over the entire sample of $n=50000$ sequences: they correspond to $G=0.35$ for the conventional method and to $G=0.82$ for the DADA method, i.e. the overall performance gap is more than twofold. As expected, the performance of both methods is nil for $\lambda_{1}=0$ and it is very sensitive to the intensity of the forcing, cf. Fig. $4 \mathrm{~b}$.

Furthermore, the skill of the DADA method is boosted when decreasing the level of model error, cf. Fig. 4c; this is an expected result, since DA becomes more reliable when the model is more accurate, and when it is known to be so. Ultimately, under perfect model conditions, i.e. as $\sigma_{Q} \rightarrow 0$, DADA reaches perfect discriminative power, with $\mathrm{G} \rightarrow 1$, no matter how small, but still positive, the forcing is; see Fig. 4d. On the other hand, the level of observational error $\sigma_{R}$ appears to have but a limited effect on DADA performance for the range of values considered, cf. Fig. 4e.

Finally, Fig. 4f shows that both methods perform better when the contrast between $p_{0}$ and $p_{1}$ is strong, but the latter does not influence the gap between the two methods, which remains nearly constant. This constant gap thus appears to quantify the additional power resulting from the extra discriminative 
features that the $\operatorname{PDF} f(\mathbf{y})$ is able to capture on top of those associated with the probability $P(\phi(\mathbf{y}) \geq u)$.

\section{Discussion and conclusions}

Considerations rooted in the causality theory of Pearl (2000) have shown that the ratio between the factual likelihood $f_{1}(\mathbf{y})$ and the counterfactual likelihood $f_{0}(\mathbf{y})$ is relevant in studying causal attribution of weather- and climaterelated events. In this paper, we described data assimilation (DA) methods and demonstrated that they are well suited for deriving $f_{0}(\mathbf{y})$ and $f_{1}(\mathbf{y})$ from trajectories in the factual and the counterfactual worlds, respectively. Besides, these methods offer the key practical advantage of being already upand-running in real time at meteorological centers.

Combining these two sets of considerations, theoretical and practical, opens a novel route towards real time, systematic causal attribution of weather- and climate-related events, thereby addressing a key challenge in the field of PEA at present (Stott et al., 2013).

\subsection{Theoretical considerations}

Implementing the DADA approach in the context of the L63 model in Section 3 allowed for a detailed step-by-step illustration of our methodological proposal. It also provided a basic test for an initial performance assessment, which showed an improved level of discriminating power with respect to the conventional approach outlined in Section 1. These results are promising, and their promise is easy to understand, given the fact that the DADA approach leverages the available information on the entire trajectory $\mathbf{y}$, as opposed to the single specific feature $\phi(\mathbf{y}) \geq u$ in the conventional approach.

It is important, though, to stress that the term "performance" here should be considered with caution: improving discriminatory performance may or may not be a desirable outcome, depending on the causal question being asked. Hannart et al. (2015) and Otto et al. (2015) have shown that the causal question being formulated reflects the subjective interests of a particular class of end-users, and that the formulation itself may dramatically affect the answer.

For example, the question "did anthropogenic $\mathrm{CO}_{2}$ emissions cause the heatwave observed over Argentina during January 2014?" has been traditionally treated by defining a "heatwave" in terms of a predefined temperature index reaching a predefined threshold, i.e., by a singular index exceeding a singular threshold. This class of questions matters for instance in the context of insurance disbursements, where a financial compensation may typically be triggered based on such an index exceedance. In this situation, the additional discriminatory power of DADA is meaningless because the DADA computation does not address the question at stake: there is simply no alternative to computing the probabilities $p_{0}$ and $p_{1}$ of the index exceeding the threshold. 
However, if the question is formulated instead as "did anthropogenic $\mathrm{CO}_{2}$ emissions cause the atmospheric conditions observed over Argentina during January 2014?" - i.e., without specifying which feature of the observed sequence is most important - then improving discrimination makes perfect sense and DADA becomes fully relevant. Furthermore, DADA is still fully relevant even if the question is formulated more specifically as "did anthropogenic $\mathrm{CO}_{2}$ emissions cause the damages generated in Argentina by the atmospheric conditions of January 2014?," provided that a model relating atmospheric observations to damages at every time step $t$ along the trajectory of the physical model used in the assimilation is available and can be integrated into the observation operator $\mathrm{H}$.

On the other hand, the results of Section 3 should also be considered with caution simply because the L63 testbed obviously differs in many respects from the real situation envisioned for future applications, both in terms of model dimension $n$ and observation dimension $d$ : in practice $n$ will be very large and $d \ll n$, while here we took $d=n=3$.

In particular, choosing a highly idealized, climatological prior distribution on the initial condition $\pi\left(\mathbf{x}_{0}\right)$ does not raise any difficulty under the tested conditions nor does it influence significantly the outcome of the procedure (not shown). The choice of $\pi\left(\mathbf{x}_{0}\right)$, however, may be an important problem in practice, when $d \ll n$, and lead to potentially spurious results.

As a consequence, it may be both necessary and useful to further constrain the so-called background PDF $\pi\left(\mathbf{x}_{0}\right)$ by using the forecasts originating from $\tau$ previous assimilation cycles, thus following the ideas of lagged-averaged forecasting (Hoffman and Kalnay, 1983; Dalcher et al., 1988). The evidence thus obtained, though, will then also depend on previous observations over the "initialization" window $[-\tau, \ldots,-1]-$ i.e., it will no longer represent exclusively the desired evidence $f(\mathbf{y})$. Besides, choosing $\tau$ optimally to constrain the initial background PDF in a satisfactory manner, while at the same time limiting the latter unwanted dependence on previous observations, is a challenging question that needs to be adressed.

More generally, the problem of evaluating the evidence $f(\mathbf{y})$ is not new in the HMM and DA literature; see, for instance, Baum et al. (1970); Hürzeler and Künsch (2001); Pitt (2002) and Kantas et al. (2009). Various algorithms are thus available to carry out this evaluation, depending on a number of key assumptions - such as lack of Gaussianity or linearity - and on the inferential setting chosen, e.g. particle filtering. These algorithms may provide accurate and effective solutions to the above problem, as well as improved alternatives to the Gaussian and linear approximation of Eq. (5), since the latter may not be sufficiently accurate for succesfully implementing the DADA approach under realistic conditions. 
4.2 Practical considerations

While we have shown here that the proposal of using DADA for event attributions has intellectual merit, its main strength lies, in our view, in down-to-earth cost considerations. By design, the DADA approach allows one to piggyback at a low marginal cost on the large and powerful infrastructures already in place at several meteorological centers, in terms of both hardware and personnel. These centers are capable of processing massive amounts of observational data with high-throughput pipelines on the world's largest computational platforms, as opposed to requiring the design, set-up and maintenance of a new and large, PEA-specific infrastructure to collect observations and generate under real time constraints - the many model simulations required by the conventional approach recalled in Section 1.

Taking a step back, it is useful to examine our proposal within the wider context of the emergence of so-called climate services. It is widely recognized that extending the scope of activity of meteorological centers from being "monoline" weather forecasting providers to becoming "multiline" climate services providers - encompassing, for instance, weather forecasting and weather event attribution as two service lines among several others - is a relevant strategic option (Hewitt et al., 2012). Such a strategy may foster the timely and cost-efficient emergence of the latter services by building upon technological and infrastructure synergies with the former. For these reasons, our proposal is particularly relevant for, and could contribute to, the implementation of the strategic option just outlined.

This being said, DADA can very well serve as a method for real time event attribution even for hypothetical climate services providers that focus uniquely or mainly on longer time scales, beyond a month, a season or a year. In such a context, DADA may allow for the assimilation of a broader range of observations, and in particular of ocean observations; it may, in fact, be important to include the latter in causal analysis when the event occurrence under scrutiny is defined over a sufficiently large time window.

Finally, it is important to remember that providing real-time attribution assessments is a major communication challenge, since different methods give different answers and different definitions of a specific event may also impact the outcome of an assessment - as mentioned above and as discussed recently by Trenberth et al. (2015). Various recent examples, such as the ongoing California drought have shown that divergences among experts may lead to confusion in the media and among stakeholders. In this respect, a detailed comparison of the DADA approach with other methods in realistic, real-time situations will be required before the method can be applied operationally.

Acknowledgements It is a pleasure to thank Fredi Otto and Dáithí Stone, who provided careful and constructive reviews of the original paper. This work has been supported by grant DADA from the Agence Nationale de la Recherche (ANR, France: AH and all coauthors) and by the Multi-University Research Initiative (MURI) N00014-12-1-0911 from the the U.S. Office of Naval Research (MG). 
Appendix A - Illustration of the computational benefit of the DADA approach. To illustrate the computational benefit, let $\mathbf{Y}$ be for instance a $d$-variate autoregressive process defined by $\mathbf{Y}_{t+1}=\mathbf{A} \mathbf{Y}_{t}+\mathbf{w}_{t}$, where $\mathbf{w}_{t}$ is an i.i.d. noise having known PDF $g(\cdot)$ and where $\mathbf{A}$ has the usual properties that insure stationarity (Gardiner, 2004). We then have:

$$
\begin{aligned}
& f(\mathbf{y})=\prod_{t=1}^{T} g\left(\mathbf{y}_{t}-\mathbf{A} \mathbf{y}_{t-1}\right) \pi\left(\mathbf{y}_{0}\right) \\
& P(\phi(\mathbf{Y}) \geq u)=\int_{\phi(\mathbf{y}) \geq u} \prod_{t=1}^{T} g\left(\mathbf{y}_{t}-\mathbf{A} \mathbf{y}_{t-1}\right) \pi\left(\mathbf{y}_{0}\right) \mathrm{d} y_{1,0} \ldots \mathrm{d} y_{d, 0} \ldots \mathrm{d} y_{d, T},
\end{aligned}
$$

with $\pi(\cdot)$ the prior PDF on the initial state $\mathbf{Y}_{0}$. Equation (7a) shows that $f(\mathbf{y})$ can be easily computed using a closed-form expression, while $P(\phi(\mathbf{Y}) \geq u)$ in Eq. (7b) is an integral on $d \times T+1$ dimensions which must instead be evaluated by using, for instance, a computationally quite costly Monte-Carlo (MC) simulation.

Appendix B - Data Assimilation. The state-estimation problem for the system given by Eqs. (4a, 4b) has an exact solution given by the following sequential Kalman filter (KF) equations:

$$
\begin{aligned}
& \mathbf{x}_{t}^{a}=\mathbf{x}_{t}^{f}+\mathbf{K}\left(\mathbf{y}_{t}-\mathbf{H} \mathbf{x}_{t}^{f}\right), \\
& \mathbf{P}_{t}^{a}=(\mathbf{I}-\mathbf{K} \mathbf{H}) \mathbf{P}_{t}^{f}, \\
& \mathbf{x}_{t+1}^{f}=\mathbf{M} \mathbf{x}_{t}^{a}, \\
& \mathbf{P}_{t+1}^{f}=\mathbf{M} \mathbf{P}_{t}^{a} \mathbf{M}^{\prime}+\mathbf{Q} .
\end{aligned}
$$

where' denotes the transpose operation. Here Eqs. (8a) and (8b) are referred to as the analysis step and denoted by a superscript $a$, while the forecast step is given by Eqs. (8c) and (8d), and is denoted by a superscript $f$ (Ide et al., 1997). The vector $\mathbf{x}_{t}^{a}$ and the matrix $\mathbf{P}_{t}^{a}$ are the mean and covariance of $\mathbf{X}_{t}$ conditional on $\left(\mathbf{Y}_{1}, \ldots, \mathbf{Y}_{t}\right)=\left(\mathbf{y}_{1}, \ldots, \mathbf{y}_{t}\right) ; \mathbf{K}=\mathbf{P}_{t}^{f} \mathbf{H}^{\prime}\left(\mathbf{H P}_{t}^{f} \mathbf{H}^{\prime}+\mathbf{R}\right)^{-1}$ is the socalled Kalman gain matrix; while $\mathbf{Q}$ and $\mathbf{R}$ are the covariances associated with $\mathbf{v}_{t}$ and $\mathbf{w}_{t}$, respectively. Following Wiener (1949), one distinguishes between filtering, in which $\mathbf{x}_{t}^{a}$ and $\mathbf{P}_{t}^{a}$ are conditioned only on the previous and current observations $\left(\mathbf{y}_{0}, \ldots, \mathbf{y}_{t}\right)$, and smoothing, in which they are conditioned on the entire sequence, $0 \leq t \leq T$. Furthermore, the sequential algorithm needs to be initialized at time $t=0$ with $\mathbf{x}_{0}^{f}$ and $\mathbf{P}_{0}^{f}$, which thus represent the a priori mean and covariance of $\mathbf{X}_{0}$, respectively, and have to be prescribed by the user.

Appendix C - Derivation of the model evidence. In this appendix, we outline the derivation of model evidence within a general Bayesian framework, and we apply the latter to the narrower KF context to obtain Eq. (5). Consider two consecutive cycles of a DA run, the first with state vector $\mathbf{x}_{t}$ and observation vector $\mathbf{y}_{t}$ at instant $t$ and the subsequent one with state vector 
$\mathbf{x}_{t+1}$ and observation vector $\mathbf{y}_{t+1}$ at instant $t+1$. We plan to find a tractable expression for the model evidence $p\left(\mathbf{y}_{t}, \mathbf{y}_{t+1}\right)$.

The model evidence provided by the full sequence of observations $\mathbf{y}=$ $\left(\mathbf{y}_{0}, \ldots, \mathbf{y}_{T}\right)$ will be inferred by recursion, using the results of this two-observation setting. In order to decouple the two cycles, one first has to spell out the Bayesian inference $p\left(\mathbf{y}_{t}, \mathbf{y}_{t+1}\right)=p\left(\mathbf{y}_{t}\right) p\left(\mathbf{y}_{t+1} \mid \mathbf{y}_{t}\right)$. We look for a tractable expression for $p\left(\mathbf{y}_{t+1} \mid \mathbf{y}_{t}\right)$ by further introducing the states $\mathbf{x}_{t+1}$ and $\mathbf{x}_{t}$ as intermediate random variables:

$$
\begin{aligned}
p\left(\mathbf{y}_{t+1} \mid \mathbf{y}_{t}\right) & =\int_{\mathbf{x}_{t+1}} p\left(\mathbf{y}_{t+1} \mid \mathbf{y}_{t}, \mathbf{x}_{t+1}\right) p\left(\mathbf{x}_{t+1} \mid \mathbf{y}_{t}\right) \mathrm{d} \mathbf{x}_{t+1} \\
& =\int_{\mathbf{x}_{t+1}} p\left(\mathbf{y}_{t+1} \mid \mathbf{x}_{t+1}\right)\left\{\int_{\mathbf{x}_{t}} p\left(\mathbf{x}_{t+1} \mid \mathbf{x}_{t}\right) p\left(\mathbf{x}_{t} \mid \mathbf{y}_{t}\right) \mathrm{d} \mathbf{x}_{t}\right\} \mathrm{d} \mathbf{x}_{t+1},
\end{aligned}
$$

where $p\left(\mathbf{y}_{t+1} \mid \mathbf{x}_{t+1}\right)$ is the likelihood of the observation vector $\mathbf{y}_{t+1}$ conditional on the state vector $\mathbf{x}_{t+1}$ and it is known from Eq. (4b). The conditional PDF $p\left(\mathbf{x}_{t} \mid \mathbf{y}_{t}\right)$ of $\mathbf{x}_{t}$ on $\mathbf{y}_{t}$ at instant $t$ - which appears on the right-hand side of the above equation - is referred to as the analysis PDF in the DA literature, where it is denoted by a superscript $a$ (Ide et al., 1997), and it constitutes the main DA output. The integral $\int_{\mathbf{x}_{t}} p\left(\mathbf{x}_{t+1} \mid \mathbf{x}_{t}\right) p\left(\mathbf{x}_{t} \mid \mathbf{y}_{t}\right) \mathrm{d} \mathbf{x}_{t}=p\left(\mathbf{x}_{t+1} \mid \mathbf{y}_{t}\right)$, in which $p\left(\mathbf{x}_{t+1} \mid \mathbf{x}_{t}\right)$ is known from the model dynamics given by Eq. (4), propagates this analysis PDF further in time, to instant $t+1$. Hence, the result of this integration coincides with the forecast PDF, denoted by superscript $f$ in the DA literature (Ide et al., 1997). It follows that this decomposition is tractable using a DA scheme that is able to estimate the conditional and forecast PDFs.

Next, let us apply the general Bayesian inference (9) to the case in which all the PDFs involved are Gaussian; this requires, in turn, that both the dynamics and observation models $\mathrm{M}$ and $\mathrm{H}$ be linear, and that the input statistics all be Gaussian. In this case, the Kalman filter allows for the exact computation of the PDFs mentioned in Eq. (9), which turn out to be Gaussian.

In the following, $\mathcal{N}(\overline{\mathbf{x}}, \mathbf{P})$ designates the Gaussian PDF of mean $\overline{\mathbf{x}}$ and covariance matrix $\mathbf{P}$. In this context, the analysis PDF at instant $t$ is $\mathcal{N}\left(\mathbf{x}_{t}^{a}, \mathbf{P}_{t}^{a}\right)$, where $\mathbf{x}_{t}^{a}$ and $\mathbf{P}_{t}^{a}$ are the analysis state and error covariance matrix at instant $t$. As a result of the linearity assumptions, the forecast PDF at instant $t+1$ is given by a Gaussian distribution $\mathcal{N}\left(\mathbf{x}_{t+1}^{f}, \mathbf{P}_{t+1}^{f}\right)$, where $\mathbf{x}_{t+1}^{f}$ and $\mathbf{P}_{t+1}^{f}$ are the forecast state and error covariance matrix at instant $t+1$. Further, the integration on $\mathbf{x}_{t+1}$ in Eq. (9) can readily be performed under these circumstances, with the outcome that $p\left(\mathbf{y}_{t+1} \mid \mathbf{y}_{t}\right)$ is distributed as $\mathcal{N}\left(\mathbf{H} \mathbf{x}_{t+1}^{f}, \mathbf{R}+\mathbf{H}_{t+1}^{f} \mathbf{H}^{\prime}\right)$.

The desired model evidence $f(\mathbf{y})$ can then be computed by recursion on successive time steps as:

$$
f(\mathbf{y})=p\left(\mathbf{y}_{0}\right) \prod_{t=1}^{T}(2 \pi)^{-\frac{d}{2}}\left|\boldsymbol{\Sigma}_{t}\right|^{-\frac{1}{2}} \exp \left\{-\frac{1}{2}\left(\mathbf{y}_{t}-\mathbf{H} \mathbf{x}_{t}^{f}\right)^{\prime} \boldsymbol{\Sigma}_{t}^{-1}\left(\mathbf{y}_{t}-\mathbf{H} \mathbf{x}_{t}^{f}\right)\right\} ;
$$

here $p\left(\mathbf{y}_{0}\right)$ represents the prior PDF of the initial state, $\boldsymbol{\Sigma}_{t}=\mathbf{R}+\mathbf{H P}_{t}^{f} \mathbf{H}^{\prime}$, and this expression coincides with Eq. (5) and can be evaluated with the help of any DA method that yields the forecast states and forecast error covariance 
matrices, such as the KF or the EnKF. Note that the traditional standard Kalman smoother would give the same result as the KF, since they share the same forecasts.

Finally, Eqs. (9) and (10) above show that the likelihood $f(\mathbf{y})$ may be obtained as a by-product of the inference on the state vector $\mathbf{x}$, which usually is the main purpose in numerical weather prediction. This idea may actually be highlighted in even greater generality by considering the equality:

$$
f(\mathbf{y})=\frac{p(\mathbf{y} \mid \mathbf{x}) p(\mathbf{x})}{p(\mathbf{x} \mid \mathbf{y})} .
$$

While Eq. (11) is a direct consequence of Bayes theorem, it also illustrates a point that is arguably not so intuitive. The likelihood $f(\mathbf{y})$ is obtained here as the ratio of two quantities: a numerator $p(\mathbf{y} \mid \mathbf{x}) p(\mathbf{x})$ that is a model premise inherently postulated by Eqs. (4a) and (4b), and a denominator $p(\mathbf{x} \mid \mathbf{y})$ that may be viewed as the end result of the primary inferxence on $\mathbf{x}$. In other words, estimating $f(\mathbf{y})$ requires only a straightforward division, provided $\mathbf{x}$ has been previously inferred.

Equation (11) thus expresses with great clarity and simplicity a fundamental idea buttressing our proposal, as it provides a general theoretical justification for the suggestion of deriving the likelihood from an inferential treatment that focuses on $\mathbf{x}$. To put it succintly, this equation basically says, "He who can do more can do less." In the context of DA, whose end purpose is to infer the state vector $\mathbf{x}$ out of an observation $\mathbf{y}$ - i.e., the more part - it is possible to obtain the likelihood as a by-product thereof - i.e., the less part - and thus almost for free.

Appendix D - PDF of the state vector. We associate a label $\omega \in \Omega$ with each realization of the random process $\mathbf{v}_{t}$ that drive the model given by Eq. (6). The PDF of the state vector $\mathbf{x}_{t}$ can be obtained as the numerical solution of the corresponding Fokker-Planck equation, and it is the mean over $\Omega$ of the sample measures obtained for each realization $\omega$ of the noise $\mathbf{v}_{t}$ and (Chekroun et al., 2011, and references therein). Each sample measure is supported on a random attractor that may have very fine structure and be time-dependent (Chekroun et al., 2011, Figs. 1-3 and supplementary material), but the PDF is supported smoothly, in the counterfactual world in which $\lambda_{0}=0$, on a "thickened" version of the fairly well-known strange attractor of the original L63 model. The latter PDF represents its attractor in dynamic system's terminology. 

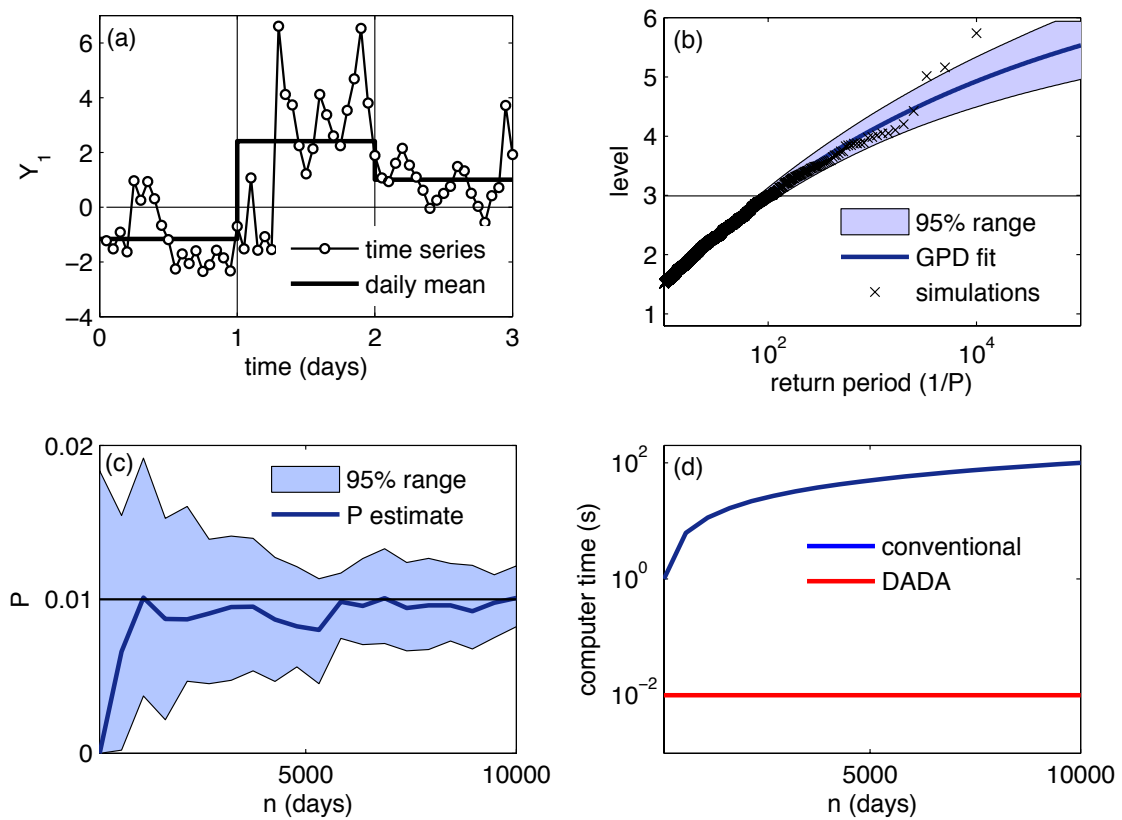

Fig. 1 Illustration of the conventional PEA approach as applied to a univariate AR(1) process. (a) Observed time series (first component $Y_{1}$, dotted line) and daily average $\phi(\mathbf{Y})$ (heavy solid line) over the three first days. (b) Threshold level (vertical axis) as a function of the return period (horizontal axis): simulated values (crosses); fit based on the Generalized Pareto distribution (GPD, heavy dark-blue line); uncertainty range at the 95\% level (light blue area); and threshold value $u=3.1$ (light solid black line). (c) Estimated value of $P=P(\phi(\mathbf{Y}) \geq u$ ) (heavy dark-blue line) using a GPD fit as a function of the sample size $n$ (horizontal axis); uncertainty range (light blue area); and true value $P=0.01$ (light solid black line). (d) Computational time on a desktop computer (seconds, vertical axis) as a function of sample size $n$ (horizontal axis) required by the conventional method (dark blue line) and the DADA method (solid red line); the latter method is explained in Sections $2 \mathrm{~b}$ and 3 below. 

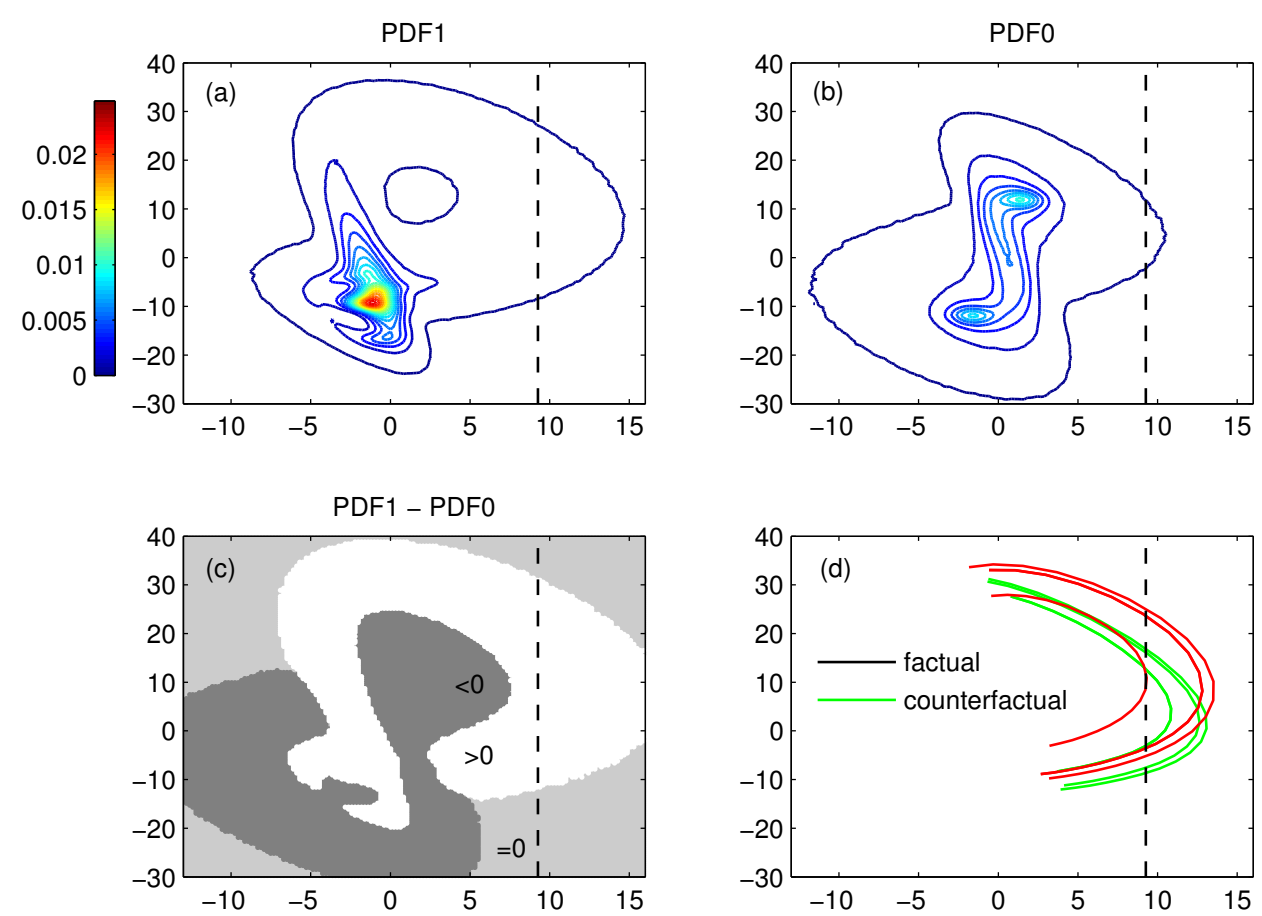

Fig. 2 Two-dimensional (2-D) projections of the PDF of the modified L63 model; the projection is onto a plane defined by the two leading eigenvectors of the factual PDF shown in the first panel. (a) PDF of the factual attractor, with $\lambda_{1}=20$ and $\sigma_{Q}=0.1$; and (b) PDF of the counterfactual attractor, with $\lambda_{0}=0$. (c) Difference between the factual and counterfactual PDFs. (d) Sample trajectories associated with an event occurrence originating from the factual (red solid lines) and counterfactual worlds (green solid lines); the vertical dashed line in all four panels indicates the threshold $u$ with respect to the horizontal axis of largest variance in the factual PDF. 

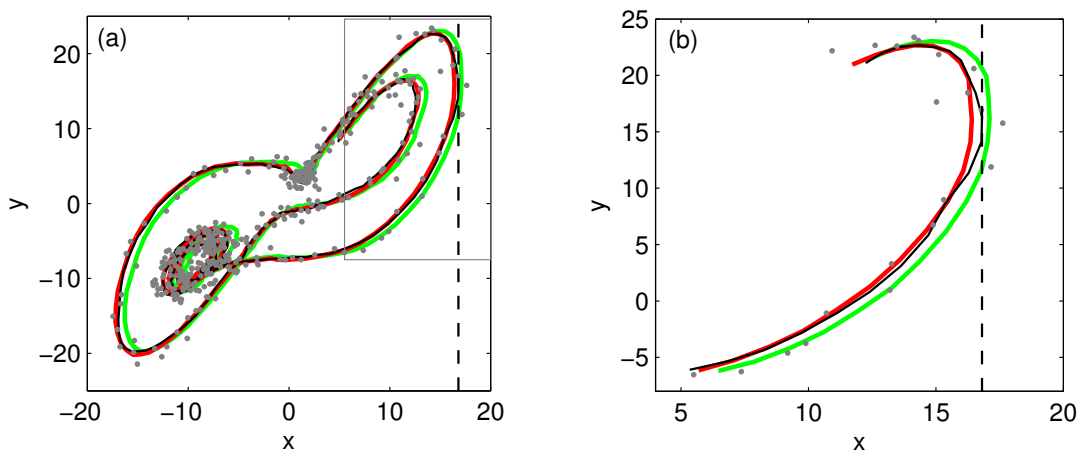

$-x^{a}$ counterfactual $-x^{a}$ factual

$y^{0}$
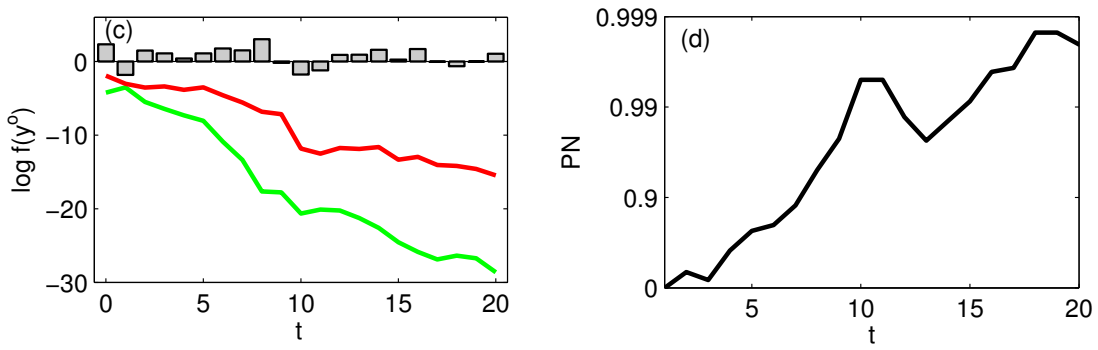

cumulative counterfactual on $[0, t]$

$\longrightarrow$ PN on $[0, t]$

cumulative factual on $[0, t]$

difference at instant $t$

Fig. 3 Sample trajectories from data assimilation (DA) in our modified L63 model. (a) True trajectory (black solid line) and the two trajectories reconstructed by DA in the factual $(i=1)$ and counterfactual $(i=0)$ worlds (red and green solid lines), respectively, over a long sequence, $T=400$; the values of $\lambda_{1}$ and $\theta_{1}$ here are the same as in Fig. 2, and the assimilated observations are shown as gray dots. (b) Same as panel (a) but zoomed over a short sequence, $T=20$. (c) Logarithm of the cumulative evidences $f_{1}(\mathbf{y})$ and $f_{0}(\mathbf{y})$ (red and green lines, respectively) computed over the window $[0, t \leq T]$; gray bars indicate the instantaneous differences between $f_{1}\left(\mathbf{y}_{t}\right)$ and $f_{0}\left(\mathbf{y}_{t}\right)$. (d) PN computed over the window $[0, t]$. 


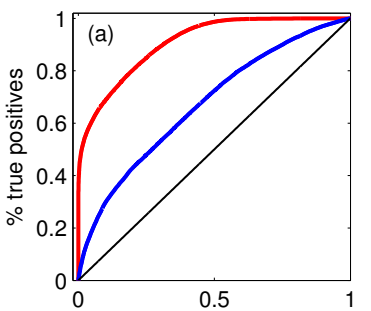

$\%$ false positives

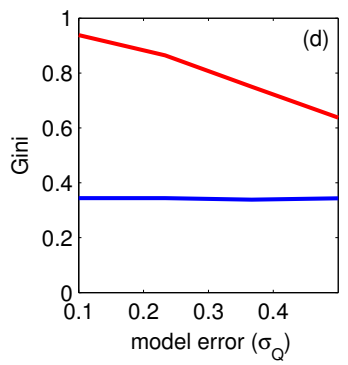

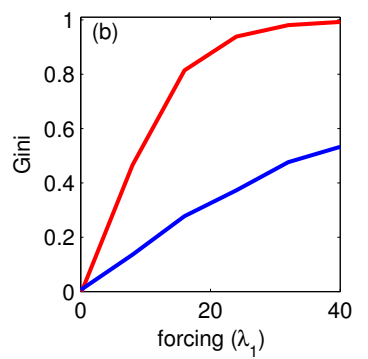
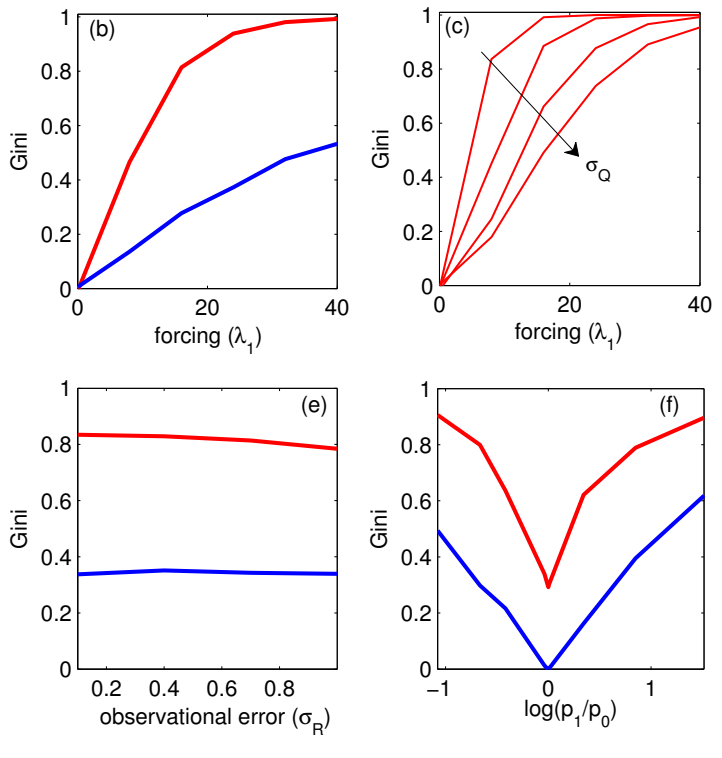

Fig. 4 Performance of the DADA and conventional methods (red vs. blue solid lines, respectively). (a) Receiver operating characteristic (ROC) curve: true positive rate as a function of false positive rate, when varying the cut-off level $u$, as obtained from the entire sample of $n=50000$ sequences; see text for details.. (b) Gini index $G$ as a function of forcing intensity $\lambda_{1}$. (c) Same as (b) for several values of $\sigma_{Q}$ and for DADA only, with the black arrow indicating the direction of growing $\sigma_{Q}$. (d) Same as (b) but as a function of model error amplitude $\sigma_{Q}$. (e) Same as (b) but as a function of observational error amplitude $\sigma_{R}$. (f) Same as (b) as a function of the logarithmic contrast between the conventional probabilities $\log p_{1} / p_{0}$. 


\section{References}

Allen M.R. (2003) Liability for climate change. Nature, 421:891-892.

Arnold L. (1998) Random Dynamical Systems. Springer, 625 pp.

Baum L.E., T. Petrie, G. Soules, N. Weiss (1970) A maximization technique occurring in the statistical analysis of probabilistic functions of Markov chains. The Annals of Mathematical Statistics, 41(1):164-171.

Balmaseda M.A., O.J. Alves, A. Arribas, T. Awaji, D.W. Behringer, N. Ferry, Y. Fujii, T. Lee, M. Rienecker, T. Rosati, D. Stammer (2009) Ocean initialization for seasonal forecasts, Oceanography Special Issue, 22(3).

Bengtsson L., M. Ghil, E. Källén (Eds., 1981) Dynamic Meteorology: Data Assimilation Methods, Springer-Verlag, New York/Heidelberg/Berlin, 330 pp.

Bhend J., J. Franke, D. Folini, M. Wild, S. Brönnimann (2012) An ensemblebased approach to climate reconstructions Clim. Past, 8:963-976.

Bocquet M., C.A. Pires, L. Wu (2010) Beyond Gaussian statistical modeling in geophysical data assimilation. Mon. Wea. Rev., 138:2997-3023.

Bocquet M. (2012) Parameter-field estimation for atmospheric dispersion: application to the Chernobyl accident using 4D-Var. Quart. J. Roy. Meteor. Soc., 138:664-681.

Bucklew J.A. (2004) Introduction to Rare Event Simulation, Springer.

Carrassi A, S. Vannitsem (2010) Model error and variational data assimilation: A deterministic formulation. Mon. Wea. Rev., 138, 3369-3386.

Carrassi A., M. Ghil, A. Trevisan, F. Uboldi (2008) Data assimilation as a nonlinear dynamical systems problem: Stability and convergence of the prediction-assimilation system. Chaos: An Interdisciplinary Journal of Nonlinear Science, 18(2):023112.

Chekroun M.D., E. Simonnet, M. Ghil, 2011: Stochastic climate dynamics: Random attractors and time-dependent invariant measures, Physica D, 240(21):1685-1700, doi :10.1016/j.physd.2011.06.005.

Chevallier F. (2013) On the parallelization of atmospheric inversions of CO2 surface fluxes within a variational framework. Geosci. Model. Dev. Discuss., $6,37-57$.

Christidis N., P.A. Stott, A. A. Scaife, A. Arribas, G. S. Jones, D. Copsey, J. R. Knight, W. J. Tennant. (2013) A New HadGEM3-A-Based System for Attribution of Weather- and Climate-Related Extreme Events. J. Clim., 26(9): 2756-2783.

Cosme E., J.M. Brankart, J. Verron, P. Brasseur, M. Krysta (2006) Implementation of a reduced-rank, square-root smoother for ocean data assimilation. Ocean Modelling, 33, 87-100.

Dalcher A., Kalnay E., Hoffman R.N. (1988) Medium-range lagged average forecasts. Mon. Wea. Rev., 116, 402-416, doi: http://dx.doi.org/10.1175/1520-0493(1988)116¡0402:MRLAF ¿2.0.CO;2.

Del Moral P., J. Garnier, Genealogical particle analysis of rare events. Ann. Appl. Probab., 15:4, 2496-2534. 
Evensen G. (2003) The ensemble Kalman filter: theoretical formulation and practical implementation. Ocean Dyn. 53:343-367.

Gardiner C. (2004) Handbook of Stochastic Methods for Physics, Chemistry and the Natural Sciences. Publisher, pls.; no web tonite.

Gelb A. (Ed.) (1974) Applied Optimal Estimation. M.I.T. Press, Cambridge, MA, 374 pp.

Ghil M., S. Childress (1987) Topics in Geophysical Fluid Dynamics: Atmospheric Dynamics, Dynamo Theory and Climate Dynamics. SpringerVerlag, New York/Berlin, 485 pp.

Ghil M., P. Malanotte-Rizzoli (1991) Data assimilation in meteorology and oceanography, Adv. Geophys., 33:141-266.

Ghil M., S. Cohn, J. Tavantzis, K. Bube, E. Isaacson (1981) Applications of estimation theory to numerical weather prediction. In: Dynamic Meteorology: Data Assimilation Methods, L. Bengtsson, M. Ghil, E. Källén (Eds.), Springer Verlag, pp. 139-224.

Gini C. (1921) Measurement of inequality of incomes. Econ. J. 31 (121):124126. doi:10.2307/2223319.

Greenland S., K.J. Rothman (1998) Measures of effect and measures of association, Chapter 4 in Rothman, K. J., Greenland, S. (eds.), Modern Epidemiology, 2nd edn., Lippincott-Raven, Philadelphia, USA.

Hannart A., J. Pearl, F.E.L. Otto, P. Naveau, M. Ghil (2015). Counterfactual causality theory for the attribution of weather and climate-related events. Bull. Am. Meteorol. Soc., in press.

Harris T. E., Kahn H. (1951). Estimation of particle transmission by random sampling. Natl. Bur. Stand. Appl. Math. Ser., 12 27-30.

Heidelberg P. (1995). Fast simulation of rare events in queueing and reliability models. ACM Trans. Modeling Computer Simulation, 5 43-85.

Hegerl G.C., O. Hoegh-Guldberg, G. Casassa, M.P. Hoerling, R.S. Kovats, C. Parmesan, D.W. Pierce, P.A. Stott (2010): Good Practice Guidance Paper on Detection and Attribution Related to Anthropogenic Climate Change. In: Meeting Report of the Intergovernmental Panel on Climate Change Expert Meeting on Detection and Attribution of Anthropogenic Climate Change [Stocker, T.F., C.B. Field, D. Qin, V. Barros, G.-K. Plattner, M. Tignor, P.M. Midgley, K.L. Ebi (eds.)]. IPCC Working Group I Technical Support Unit, University of Bern, Bern, Switzerland.

Hewitt C., S. Mason, D. Walland (2012) The Global Framework for Climate Services, Nature Climate Change, 2, 831-832.

Hoffman R.N., Kalnay, E. (1983) Lagged average forecasting, an alternative to Monte Carlo forecasting. Tellus, 35A, 100-118, doi: 10.1111/j.16000870.1983.tb00189.x.

Houtekamer P.L., H.L. Mitchell, G. Pellerin, M. Buehner, M. Charron (2005) Atmospheric data assimilation with an ensemble Kalman filter: Results with real observations. Mon. Wea. Rev., 133, 604-620.

Hume D. (1748) An Enquiry Concerning Human Understanding. Reprinted by Open Court Press (1958), LaSalle, IL, USA. 
Hürzeler M., Künsch H.R. (2001) Approximation and maximising the likelihood for a general state-space model. In: Sequential Monte Carlo Methods in Practice [Doucet, A., De Freitas, J.F.G., Gordon N.J. (eds.)]. SpringerVerlag, New York, USA.

Ide K., P. Courtier, M. Ghil, A. Lorenc (1997) Unified notation for data assimilation: Operational, sequential and variational. J. Meteor. Soc. Japan, 75:181-189.

Ihler A.T., S. Kirshner, M. Ghil, A.W. Robertson, P. Smyth (2007) Graphical models for statistical inference and data assimilation. Physica D, 230, 72-87, 2007.

IPCC (2013) Summary for Policymakers. In: Climate Change 2013: The Physical Science Basis. Contribution of Working Group I to the Fifth Assessment Report of the Intergovernmental Panel on Climate Change [Stocker, T.F., D. Qin, G.-K. Plattner, M. Tignor, S.K. Allen, J. Boschung, A. Nauels, Y. Xia, V. Bex and P.M. Midgley (eds.)]. Cambridge University Press, Cambridge, United Kingdom and New York, NY, USA.

Jazwinski A.H. (1970) Stochastic and Filtering Theory. Mathematics in Sciences and Engineering Series, Vol. 64. Academic Press, 376 pp.

Kalman R.E. (1960) A new approach to linear filtering and prediction problems. J. Basic Eng., 82D:33-45.

Kalnay E. (2002) Atmospheric Modeling, Data Assimilation and Predictability, Cambridge University Press, Cambridge, UK.

Kantas N., A. Doucet, S.S. Singh, J.M. Maciejowski (2009) An overview of sequential Monte Carlo methods for parameter estimation. In: General StateSpace Models, IFAC System Identification, no. Ml.

Kondrashov D., C.J. Sun, M. Ghil (2008) Data assimilation for a coupled ocean-atmosphere model. Part II: Parameter estimation. Mon. Wea. Rev., 136, 50625076, doi: 10.1175/2008MWR2544.1.

Kondrashov D., Y. Shprits, M. Ghil (2011) Log-normal Kalman filter for assimilating phase-space density data in the radiation belts. Space Weather, 9, S11006, doi:10.1029/2011SW000726.

Le Dimet F.X., O. Talagrand (1986) Variational algorithms for analysis and assimilation of meteorological observations: Theoretical aspects. Tellus, 38 A:97-110.

Lee T.C.K., F.W. Zwiers, M. Tsao (2008) Evaluation of proxy-based millennial reconstruction methods. Climate Dyn., 31, 263-281.

Lorenz E.N. (1963) Deterministic non-periodic flow. J. Atmos. Sci. 20:130141.

Lorenz M.O. (1905) Methods of measuring the concentration of wealth. Publications of the American Statistical Association, 9 (70): 209219, doi:10.2307/2276207.

Martin M.J. et al. (2014) Status and future of data assimilation in operational oceanography. J. of Oper. Ocean., in press.

Massey N., Jones R., Otto F.E.L., Aina T., Wilson S., Murphy J.M., Hassell D., Yamazaki Y.H., Allen M.R. (2014) weather@home - development and validation of a very large ensemble modelling system for probabilistic event 
attribution. Q. J. R. Meteorol. Soc. doi: 10.1002/qj.2455

Otto F.E.L., Boyd, E., Jones, R.G., Cornforth, R.J., James, R., Parker, H.R., Allen, M.R. (2015) Attribution of extreme weather events in Africa: a preliminary exploration of the science and policy implications. Climatic Change. Palmer T.N. (1999) A non-linear dynamical perspective on climate prediction. J. Clim. 12:575-591.

Pearl J. (2000) Causality: Models, Reasoning and Inference, Cambridge University Press, Cambridge, United Kingdom and New York, NY, USA.

Pitt M.K. (2002) Smooth particle filters for likelihood evaluation and maximisation. Warwick Economic Research Papers, No. 651.

Robert C., E. Blayo, J. Verron (2006) Comparison of reduced-order sequential, variational and hybrid data assimilation methods in the context of a Tropical Pacific ocean model. Ocean Dynamics, 56, 624-633.

Roques L., M.D. Chekroun, M. Cristofol, S. Soubeyrand, M. Ghil (2014) Parameter estimation for energy balance models with memory. Proc $R$. Soc. A, 470, 20140349.

Ruiz J., M. Pulido, T. Miyoshi (2013) Estimating model parameters with ensemble-based data assimilation: A review. JMSJ, 91, 2, 79-99.

Sakov P., Counillon F., Bertino L., Lister K.A., Oke P.R., Korablev A. (2012) TOPAZ4: an ocean-sea ice data assimilation system for the North Atlantic and Arctic, Ocean Sci., 8, 633-656, doi:10.5194/os-8-633-2012.

Stone D.A., M.R. Allen (2005) The end-to-end attribution problem: from emissions to impacts. Clim. Change, 71:303-318.

Stott P.A., et al. (2013) Attribution of weather and climate-related events, in Climate Science for Serving Society: Research, Modelling and Prediction Priorities, G.R. Asrar and J. W. Hurrell (Eds.), Springer, in press.

Stott P.A., Stone D.A., Allen M.R. (2004) Human contribution to the European heatwave of 2003. Nature, 432:610-614.

Talagrand O. (1997) Assimilation of observations, an introduction, J. Meteor. Soc. Japan, 75 (1B):191-209.

Tandeo P., Pulido M., Lott F. (2014), Offline parameter estimation using EnKF and maximum likelihood error covariance estimates: Application to a subgrid-scale orography parametrization. Q. J. R. Meteorol. Soc. doi: $10.1002 /$ qj.2357

Trenberth, K. E., Fasullo, J. T., Shepherd, T. G. (2015) Attribution of climate extreme events. Nature Clim. Change 5, 725-730.

Wiener N. (1949) Extrapolation, Interpolation and Smoothing of Stationary Time Series, with Engineering Applications. M.I.T. Press, Cambridge, MA, $163 \mathrm{pp}$.

Wouters J. and F. Bouchet (2015) Rare event simulation of the chaotic Lorenz 96 dynamical system. Geophysical Research Abstracts, EGU General Assembly 2015 Vol. 17, EGU2015-10421-1. 